\title{
Childhood Psychosocial Determinants of Cardiovascular Health
}

Samuel S. Gidding

Thomas Jefferson University

Erica D. Sood

Thomas Jefferson University

Follow this and additional works at: https://jdc.jefferson.edu/medfp

Part of the Cardiology Commons, and the Pediatrics Commons Let us know how access to this document benefits you

\section{Recommended Citation}

Gidding, Samuel S. and Sood, Erica D., "Childhood Psychosocial Determinants of Cardiovascular Health" (2016). Department of Medicine Faculty Papers. Paper 163.

https://jdc.jefferson.edu/medfp/163

This Article is brought to you for free and open access by the Jefferson Digital Commons. The Jefferson Digital Commons is a service of Thomas Jefferson University's Center for Teaching and Learning (CTL). The Commons is a showcase for Jefferson books and journals, peer-reviewed scholarly publications, unique historical collections from the University archives, and teaching tools. The Jefferson Digital Commons allows researchers and interested readers anywhere in the world to learn about and keep up to date with Jefferson scholarship. This article has been accepted for inclusion in Department of Medicine Faculty Papers by an authorized administrator of the Jefferson Digital Commons. For more information, please contact: JeffersonDigitalCommons@jefferson.edu. 


\section{Childhood Psychosocial Determinants of Cardiovascular Health}

Erica Sood, $\mathrm{PhD}^{1,2,3}$ and Samuel S. Gidding, $\mathrm{MD}^{1,3}$

${ }^{1}$ Nemours Cardiac Center, Alfred I. duPont Hospital for Children, Wilmington, DE.

${ }^{2}$ Nemours Neurosciences Center, Alfred I. duPont Hospital for Children, Wilmington, DE.

${ }^{3}$ Sidney Kimmel Medical College, Thomas Jefferson University, Philadelphia, PA.

Street Address:

Nemours Cardiac Center

Alfred I. duPont Hospital for Children

1600 Rockland Road

Wilmington, DE 19803

Phone: 302-651-6600

Fax: 302-651-5345

erica.sood@nemours.org (corresponding author)

samuel.gidding@nemours.org

Keywords: Psychosocial; Social Determinants of Health; Childhood Risk Factors; Ideal Cardiovascular Health; Cardiovascular Risk; Cardiovascular Diseases. 


\begin{abstract}
Understanding risk and protective factors that impact cardiovascular health is of utmost importance. There is ample evidence that cardiovascular health begins in childhood, tracks over time, and is subject to adverse social influences. This paper reviews key studies examining the relations of psychosocial factors in childhood to cardiovascular health in adulthood. The existing literature provides evidence for both individual and cumulative effects of childhood psychosocial factors on adult cardiovascular health across the population, although the specific mechanisms underlying these relationships are not yet fully understood. This paper also includes a discussion of evidence-based strategies for prevention and treatment of childhood psychosocial problems. The extent to which these programs lead to improved cardiovascular health in high-risk groups or across the population by impacting psychosocial factors has not yet been studied, but is a clear future direction for research and policy.
\end{abstract}




\section{Introduction}

The American Heart Association (AHA) impact goals for 2020 emphasize not only the reduction of cardiovascular events, but also the promotion of cardiovascular health across the entire population [1]. Ideal cardiovascular health is defined by the presence of four ideal health behaviors (nonsmoking, body mass index $<25 \mathrm{~kg} / \mathrm{m}^{2}$, physical activity at goal levels, and pursuit of a diet consistent with current guideline recommendations) and three ideal health factors (untreated total cholesterol $<200 \mathrm{mg} / \mathrm{dL}$, untreated blood pressure $<120 /<80 \mathrm{~mm} \mathrm{Hg}$, and fasting blood glucose $<100 \mathrm{mg} / \mathrm{dL})$. While studies support the inverse relationship between ideal cardiovascular health and cardiovascular morbidity and mortality, the prevalence of ideal cardiovascular health in the United States population is extremely low [2, 3].

The purpose of the current paper is to review what is currently known about the relations of psychosocial factors in childhood to cardiovascular health in adulthood. There is ample evidence that cardiovascular health begins in childhood, tracks over time, and is subject to adverse social influences $[1,4 \bullet \bullet$. This is not meant to be an exhaustive review of the literature, but instead an opportunity to highlight key population studies in which psychosocial factors were measured prospectively during childhood and cardiovascular health was measured in adulthood utilizing a comprehensive outcome variable (i.e., ideal cardiovascular health, clustered metabolic risk) (Table 1). This paper also includes a discussion of evidence-based strategies for prevention and treatment of childhood psychosocial problems, as these strategies could potentially improve cardiovascular outcomes through their effects on psychosocial health.

\section{What are Psychosocial Factors?}

While psychosocial factors are commonly discussed in medical literature and practice, there is no one clear definition of the term psychosocial and all that it encompasses. Instead, it 
has become an umbrella term for the multitude of factors that are not biologically measurable and through which diverse research inquiries are carried out [5]. Oxford English Dictionary defines psychosocial as "of or relating to the interrelation of social factors and individual thought and behavior" [6]. This broad definition suggests that any construct involving how people think, feel and act within the context of their social environment can be considered psychosocial. Because children are raised in an environment impacted by the economic resources, health behaviors, and social-emotional functioning of parents and family, many studies examining child health outcomes also group these constructs under the term psychosocial. To be consistent with the use of this term across the studies reviewed in this paper, psychosocial will be defined broadly to include parental and family socioeconomic, health and emotional factors that could impact child health and wellbeing.

\section{Review of Key Studies}

\section{Cardiovascular Risk in Young Finns Study}

The Cardiovascular Risk in Young Finns study is a prospective, longitudinal study assessing risk factors and precursors for cardiovascular disease (CVD) [7]. The baseline survey was conducted in 1980 with 3596 children and adolescents (ages 3 - 18) across Finland. Six childhood psychosocial factors proposed to be important aspects of childhood psychosocial environment based on prior literature, namely socioeconomic environment, family emotional environment, parental health behaviors, stressful events, self-regulation of the child, and social adjustment of the child, have been identified from parent-report items included in the baseline survey. The adulthood assessment, which includes measurement of ideal cardiovascular health, was conducted in 2007 when participants were $30-45$ years of age $(\mathrm{N}=1089$ after excluding those with type 1 diabetes at baseline or missing data) [8•]. 
Recent analyses conducted with this cohort support relationships between individual and cumulative psychosocial factors in childhood and ideal cardiovascular health in adulthood [8•]. Favorable socioeconomic environment, family emotional environment, health behavior in the family and child self-regulatory behavior were found to be independent predictors of ideal cardiovascular health in adulthood after accounting for age, sex, adult cardiac medication use, and known childhood cardiovascular risk factors. However, only favorable socioeconomic environment and child self-regulatory behavior remained independent predictors when examined in conjunction with the other psychosocial factors. A greater number of favorable psychosocial factors in childhood resulted in more ideal cardiovascular health in adulthood, and this relationship operated across the whole gradient of cardiovascular health, from those meeting few to those meeting many or all ideal cardiovascular health metrics. Children with the most psychosocial advantages had an ideal cardiovascular health index in adulthood that was almost one point higher than those with the least psychosocial advantages, which is comparable to attaining a favorable level on any one of the seven components that comprise the ideal cardiovascular health index. Of note, for several of the psychosocial indices (including selfregulatory behavior), the vast majority of the cohort achieved the highest possible score, indicating that those at highest risk for poor cardiovascular health likely represented the extreme adverse end of the population distribution and may have many proximate problems to worry about other than CVD 30-50 years in the future.

Individual and cumulative effects of childhood psychosocial factors (socioeconomic status and parental smoking) on ideal cardiovascular health have been further demonstrated by analyses with the Young Finns data in conjunction with data from population-based studies in Australia (Childhood Determinants of Adult Health Study; CDAH) and the United States 
(Princeton Follow-up Study) [9•]. After accounting for age, sex, race and known childhood cardiovascular risk factors, socioeconomic status continued to predict ideal cardiovascular health in adulthood in all three cohorts, whereas parental smoking predicted ideal cardiovascular health in the Young Finns cohort only. The associations between childhood socioeconomic status and adult ideal cardiovascular health generally persisted when adjusting for participants' own socioeconomic status in adulthood. Participants in the Young Finns and CDAH cohorts who had both childhood risk factors (low socioeconomic status and exposed to parental smoking) had 0.4-0.6 adult ideal metrics less than those participants without these risk factors, which the authors estimated could equate to 15-25\% higher incidence of CVD.

\section{Collaborative Perinatal Project}

The Collaborative Perinatal Project enrolled pregnant women in the United States between 1959 and 1966, followed by frequent assessment of women and their offspring from birth to age seven [10]. Three childhood psychosocial factors, namely attention regulation capacity, cognitive ability, and positive home environment, have been identified through a combination of observational ratings, standardized testing results, and parent-report items. A subsample of the now adult offspring from the New England sites have undergone assessment through two follow up studies (Brown-Harvard Transdisciplinary Tobacco Use Research Center, EdHealth study), which included measurement of Favorable Cardiovascular Risk, defined as meeting the following criteria in midlife: systolic blood pressure $\leq 120 \mathrm{~mm} \mathrm{Hg}$, diastolic blood pressure $\leq 80 \mathrm{~mm} \mathrm{Hg}$, not taking antihypertensive medication, total cholesterol $<200 \mathrm{mg} / \mathrm{dL}$, not taking cholesterol-lowering medication, body mass index $<25 \mathrm{~kg} / \mathrm{m}^{2}$, not having diabetes mellitus, and nonsmoker [11•]. 
Recent analysis of 415 adults (mean age of 42.2 years) with the relevant baseline and follow-up data supports relationships between individual and cumulative psychosocial factors in childhood and favorable cardiovascular health in adulthood [11•]. Adjusting for age, race and childhood factors (which included childhood cardiovascular health as well as family socioeconomic status), higher levels of childhood attention regulation capacity, cognitive ability, and positive home environment were associated with a greater likelihood of favorable cardiovascular risk in adulthood. The association with childhood attention regulation was maintained when also accounting for adulthood factors (education attainment, depressive symptoms, physical activity, and diet), whereas these adulthood factors appeared to be on the pathway from childhood cognitive ability and positive home environment to adult cardiovascular risk. The effects of childhood psychosocial factors were cumulative as those with high levels of each childhood factor had approximately four times higher odds of favorable cardiovascular risk in adulthood as compared to those low in all factors.

\section{Dunedin Multidisciplinary Health and Development Study}

The Dunedin Multidisciplinary Health and Development Study is a longitudinal investigation of health and behavior among individuals born between April 1972 and March 1973 in Dunedin, New Zealand [12]. Exposure to adverse childhood experiences, namely childhood low socioeconomic status, maltreatment, and social isolation, were assessed at various time points during the first 15 years of life through a combination of observational ratings and parent- and teacher-report items. The 32-year follow up assessment was completed by 972 participants and included measurement of clustered metabolic risk, defined as three or more of the following: overweight, high blood pressure, high total cholesterol, low high-density 
lipoprotein cholesterol, high glycated hemoglobin, and low maximum oxygen consumption levels adjusted for body weight [13].

Analyses conducted with this cohort support relationships between individual and cumulative psychosocial factors in childhood and clustering of metabolic risk markers at age 32 years [13]. After controlling for family history of heart disease and high childhood body mass index, children growing up in socioeconomically disadvantaged families and those who scored very high in social isolation were both at greater risk for metabolic risk marker clustering in adulthood. The risk of developing metabolic risk marker clustering increased from those with no history of any of the three adverse childhood experiences to those with a history of two or more adverse childhood experiences in a dose-response fashion.

\section{Summary of Observational and Longitudinal Data}

The existing literature provides evidence for a cumulative effect of childhood psychosocial factors on adult cardiovascular health. This effect appears to operate across the entire population, indicating that the promotion of childhood psychosocial health is likely to be beneficial for those at lower risk for CVD in addition to those at highest risk. Three psychosocial constructs in particular have demonstrated robust associations with adult cardiovascular health across multiple prospective studies: family socioeconomic status, family emotional environment, and childhood self-regulation (encompassing attention regulation, frustration tolerance, and aggression control). Several additional constructs, including parental smoking and child social isolation, predicted adult cardiovascular health in a single prospective study and require further investigation.

\section{Strengths and Limitations of Existing Literature}


The reviewed studies have several strengths, including the use of large, population-based samples across multiple continents, prospective assessment of childhood psychosocial factors, and evaluation of both individual and cumulative effects on adult cardiovascular health. However, they are also subject to methodological limitations common among longitudinal, population-based cohort studies. Measurement of psychosocial constructs was limited to items chosen for inclusion decades ago, which in most cases did not reflect the validated tools now widely available. Because population-based studies must balance breadth versus depth and psychosocial factors were not the primary focus of study, few items assessed each construct (sometimes as few as one), often relying on parent self-report or report of the child. Relatedly, measurement of the same or similar constructs varied widely across studies. Follow up assessments were additionally subject to attrition and differential participation related to the aims of the follow up study, which may impact generalizability of results.

\section{Impact of Population Trends on Psychosocial Stress}

The prevalence of psychosocial stress is tightly linked to larger trends in the general population, particularly with regard to education, poverty and disparities. Changes to the prevalence of various psychosocial factors included in the reviewed studies, which were initiated decades ago, may reduce generalizability of results to the current era. As previously mentioned, the vast majority of the Young Finns cohort achieved the highest possible score for several of the psychosocial indices, indicating that those at highest risk for poor cardiovascular health represented the extreme adverse end of the population distribution across Finland in 1980 [8•]. This pattern would likely not generalize to the current United States population, which has experienced a concerning trend towards increased poverty and disparities [14]. Median income is falling among the lower deciles of income while rising among the top decile [14]. Those living in 
poverty or in lower classes are much less likely to receive an education comparable with the upper class [15]. Over $40 \%$ of United States children are born to unmarried women and low birth weight rates have increased for years, plateauing at about $8 \%$ [16].

These factors, combined with the ongoing obesity epidemic (itself related to some of the factors listed above), may contribute to lack of improvement in CVD and all cause mortality in young to middle aged adults. Recent adverse trends in young and middle aged adult cardiovascular morbidity and mortality, particularly in women, have been observed with those less than 55 years of age not sharing in the significant reductions in cardiovascular events observed in older individuals [17]. Higher all cause mortality rates have also been observed in white United States American males between 45 and 54 years of age [18]. United States adolescents have the highest mortality rates in the world mostly due to violence, suicide and traffic accidents [19].

\section{Future Directions}

Contemporary prospective cohort studies are needed. These studies should be specifically designed to investigate the cardiovascular impact of childhood psychosocial stressors prevalent in the current era using gold standard validated measures. Such studies should also aim to elucidate the psychological, behavioral and biological mechanisms by which psychosocial factors individually and cumulatively impact cardiovascular health, which to date has been mostly studied through cross-sectional research with adults $[4 \bullet \bullet]$. One theory of particular relevance to early development (and to the increased prevalence of low birth weight in the United States population) is the fetal origins hypothesis, which asserts that the fetus adapts to an abnormal environment such as malnutrition by altering cell programming at a critical period in development [20]. This theory has been supported by studies demonstrating relationships 
between low birth weight in term infants and increased risk of later atherosclerosis, type 2 diabetes mellitus, systemic hypertension, and metabolic syndrome [4••, 21, 22, 23, 24].

\section{Supporting Psychosocial Health: Family-Focused Preventive Programs}

Despite the need for additional longitudinal research in this area, it is not necessary or appropriate to wait 30 years to promote cardiovascular health by intervening on psychosocial risk factors. To date, cardiovascular health promotion and prevention efforts have focused predominantly on modifying health behaviors, such as smoking, physical activity, and diet [1]. Targeting psychosocial factors in childhood with the specific goal of improving adult cardiovascular health has not been a primary area of clinical or research focus. However, there is a growing recognition of its importance, as evidenced by a recent AHA Scientific Statement asserting that "the most significant opportunities for reducing death and disability from CVD in the United States lie with addressing the social determinants of cardiovascular outcomes" [4••].

Many evidence-based programs exist, ranging from population-wide preventive interventions ("universal prevention") to preventive interventions for individuals at risk for developing psychosocial problems ("selective prevention") to interventions for those already exhibiting problems (“treatment”). These programs also differ in setting, mode of delivery, duration/intensity and targeted developmental stage. Three program models will be reviewed as examples: Nurse-Family Partnership (NFP), Family Check-Up (FCU) and Incredible Years ${ }^{\circledR}$ (IY). Comprehensive information on a wide range of child- and family-focused prevention and treatment programs are described elsewhere [25, 26, 27].

\section{Home Visiting Programs: Nurse-Family Partnership and Family Check-Up}

Several home visiting programs have met the US Department of Health and Human Services criteria for evidence of effectiveness [27]. These programs are delivered in-home to 
pregnant women and/or parents to promote skills needed to raise children who are physically, socially and emotionally healthy, support parental health and economic self-sufficiency, and connect families with available resources. Many target only those families at highest risk, whereas others are available to all families with higher intensity services reserved for those with specific risk factors.

\section{Nurse-Family Partnership}

NFP serves low income, first-time mothers from pregnancy until the child turns two through weekly or every other week home visits by a registered nurse trained in the NFP model. Program goals include improving prenatal health and outcomes, child health and development, and family economic self-sufficiency and maternal life course development. Long-term follow up of three randomized controlled trials conducted in New York in the 1970s and in Tennessee and Colorado in the 1990s indicate positive effects on a variety of child and family outcomes, including prenatal health, family socioeconomic status (e.g., increased maternal employment, reduced use of welfare and food stamps), family environment (e.g., increased paternal involvement, reduced maternal arrests and convictions), and child behavior and substance use $[28,29,30]$. NFP has been adopted by the majority of U.S. states and has demonstrated a substantial return on investment [29]. The impact of NFP on the cardiovascular health of children (now young adults) who received the intervention has not been examined to our knowledge, although a recent report suggests that NFP may reduce the likelihood of maternal death from all causes, including diabetes, endocarditis, pulmonary embolism and stroke [31]. Certainly, this seems like an area of opportunity for research examining the impact of a widely available maternal and child public health program on adult cardiovascular health. The focus on 
prenatal health and outcomes may be particularly relevant, given research linking intrauterine growth retardation and low birth weight to adult CVD [21, 22, 23].

\section{Family Check-Up}

FCU serves families of children two to 17 years of age who have specific risk factors such as socioeconomic disadvantage, maternal depression, or child academic failure. Program goals include reducing children's emotional, behavioral and academic problems and improving maternal depression, parental involvement, and positive parenting. The "check-up" refers to a series of three home-visits, often repeated on a yearly basis, consisting of an initial interview, comprehensive assessment of parenting and child behaviors, and feedback including discussion of family strengths and possible areas of change using a motivational interviewing approach [32]. Interventions following the "check-up" are tailored to the family's particular strengths and needs and may include more intensive parenting support designed to enhance parents' skills in positive behavior support, healthy limit-setting, and relationship-building. Follow up studies of children and adolescents whose families participated in FCU report increased school readiness, improved emotional and behavioral functioning and reduced substance use $[33,34,35]$. There is also preliminary evidence that FCU may have an indirect effect on later weight gain and obesity $[36,37]$. No studies to our knowledge have examined the impact of FCU on other components of ideal cardiovascular health.

\section{Group Intervention Programs: Incredible Years $^{\circledR}$}

IY is a set of interlocking group-based programs serving children, parents, and teachers [38]. Program goals include preventing and treating behavior problems and promoting social, emotional, and academic competence for children birth to age 12. Program curriculum and duration varies depending on child age and level of need and includes brief "universal" programs 
as well as more intensive programs for children with diagnosed developmental or mental health problems and their parents and teachers. Follow up studies of IY report improvements in positive parenting, child behavior and school readiness, among other positive outcomes [38, 39]. There is also preliminary evidence that IY parent and child groups may result in positive child health outcomes approximately five years later, including lower body mass index and blood pressure, more physical activity, less "screen time" and smaller percent of calories from carbohydrates [40], despite these not being the focus of intervention. The mechanism by which these programs may impact child health outcomes and the extent to which positive outcomes persist to adolescence and adulthood have not yet been studied, but are certainly areas of future direction.

\section{Moderators of Outcome}

Unfortunately, existing preventive interventions don't work for every family. Metaanalyses of various parent-training interventions aimed at modifying parenting and child problem behavior have identified specific psychosocial risk factors, including parental depression, low income, and single parent status, associated with poorer outcomes [41, 42]. However, more promising results have been found for programs that specifically target common barriers to intervention engagement and retention among socioeconomically disadvantaged families (i.e., delivering interventions in the home; incorporating goals and support related to family economic self-sufficiency; incorporating financial or material incentives) [43, 44]. In a trial of 731 lowincome toddlers randomly allocated to the in-home FCU intervention versus no intervention, effects on child problem behavior were not moderated by parental depression, parental marital or drug problems, or having a mother who had given birth as a teenager, suggesting that children with these disadvantages were just as likely to do well following intervention [43]. The only identified psychosocial risk factor associated with poorer intervention response was single parent 
status, whereas lower maternal education was actually associated with greater improvement in child problem behavior. In a trial of 153 preschoolers from socially disadvantaged neighborhoods randomly allocated to IY intervention versus waitlist control, effects on child problem behavior were not moderated by very low income (compared to the average for these low income areas), single parent status, or having a mother who had given birth as a teenager, whereas children of more depressed mothers fared better following intervention, relative to children in the control group, who had much poorer behavioral outcomes when their mother was depressed [44]. Although the IY intervention was delivered in the community, home visits were made to parents who missed sessions and meals, daycare and transport were provided.

Despite promising results for those who participate, it is a reality that not all families choose to enroll in available preventive interventions. Even in the case of a brief, three-session intervention delivered in the home for which families were given a financial incentive for participation, $17 \%$ of at-risk families chose not to enroll [43]. Families who decline interventions despite psychosocial risk factors may unfortunately be those in greatest need of assistance, such as first-time parents lacking knowledge regarding the challenges of child rearing or those uncomfortable with the model of in-home visitation (possibly due to unsuitable or unstable living conditions) [45]. Clearly, continued efforts to access and engage families at risk of nonparticipation and attrition are needed, with the understanding that barriers to participation are not static and will likely shift over time along with population trends in education, poverty and disparities.

\section{Role of the Medical Provider}

Medical providers serving pregnant women, children and families are encouraged to familiarize themselves with available prevention/intervention programs that have proven success 
in improving psychosocial health and reducing sociodemographic risk factors. While it is typically not the role of the obstetrician, pediatrician or family doctor to provide the psychosocial intervention, they are responsible for identifying risk factors that may lead to poor health outcomes and making referrals to the appropriate supports. Brief standardized screening tools assessing family psychosocial risk and child social-emotional functioning are available for use in medical settings and can help to identify those children and families in need of universal or selective preventive interventions or treatment $[46,47]$. The integration of psychologists, social workers and other mental health providers into pediatric and family medical practices can further enhance the process of psychosocial screening, consultation and referral, with the ultimate goal of promoting both psychosocial and physical health [48].

\section{Conclusions}

Psychosocial factors in childhood appear to impact adult cardiovascular health, although the specific mechanisms underlying this relationship are not yet fully understood. There are numerous existing evidenced-based programs known to promote child and family psychosocial health. The extent to which these programs lead to improved cardiovascular health in high-risk groups or across the population by impacting psychosocial factors has not yet been studied, but is a clear future direction for research and policy. 


\section{References}

1. Lloyd-Jones DM, Hong Y, Labarthe D, et al. Defining and setting national goals for cardiovascular health promotion and disease reduction: the American Heart Association's strategic Impact Goal through 2020 and beyond. Circulation. 2010;121:586-613.

2. Bambs C, Kip KE, Dinga A, Mulukutla SR, Aiyer AN, Reis SE. Low prevalence of "ideal cardiovascular health" in a community-based population: the heart strategies concentrating on risk evaluation (Heart SCORE) study. Circulation. 2011;123:850-7.

3. Folsom AR, Yatsuya H, Nettleton JA, Lutsey PL, Cushman M, Rosamond WD; ARIC Study Investigators. Community prevalence of ideal cardiovascular health, by the American Heart Association definition, and relationship with cardiovascular disease incidence. J Am Coll Cardiol. 2011;57:1690-6.

4. ••Havranek EP, Mujahid MS, Barr DA, et al. Social determinants of risk and outcomes for cardiovascular disease: a scientific statement from the American Heart Association. Circulation. 2015;132:873-98. This scientific statement summarizes the current state of knowledge regarding the influence of social factors on the incidence, treatment, and outcomes of cardiovascular diseases.

5. Martikainen P, Bartley M, Lahelma E. Psychosocial determinants of health in social epidemiology. Int J Epidemiol. 2002;31:1091-3.

6. Oxford Dictionaries. Oxford University Press. http://www.oxforddictionaries.com/us/definition/american_english/psychosocial. Accessed January 12, 2016.

7. Raitakari OT, Juonala M, Rönnemaa T, et al. Cohort profile: the Cardiovascular Risk in Young Finns Study. Int J Epidemiol. 2008;37:1220-6. 
8. •Pulkki-Råback L, Elovainio M, Hakulinen C, et al. Cumulative effect of psychosocial factors in youth on ideal cardiovascular health in adulthood: the Cardiovascular Risk in Young Finns Study. Circulation. 2015;131:245-53. This study provides support for relationships between individual and cumulative psychosocial factors in childhood and ideal cardiovascular health in adulthood.

9. -Laitinen TT1, Pahkala K, Venn A, et al. Childhood lifestyle and clinical determinants of adult ideal cardiovascular health: the Cardiovascular Risk in Young Finns Study, the Childhood Determinants of Adult Health Study, the Princeton Follow-Up Study. Int J Cardiol. 2013;169:126-32. This study provides support for the relationship between socioeconomic status in childhood and ideal cardiovascular health in adulthood using data from three population-based cohorts in Finland, Australia and the United States.

10. Niswander KR, Gordon M. The Collaborative Perinatal Study of the National Institute of Neurological Diseases and Stroke: The Women and Their Pregnancies. W.B. Saunders Company; Philadelphia: 1972.

11. Appleton AA, Buka SL, Loucks EB, Rimm EB, Martin LT, Kubzansky LD. A prospective study of positive early-life psychosocial factors and favorable cardiovascular risk in adulthood. Circulation. 2013;127:905-12. This study provides support for the relationships between individual and cumulative psychosocial factors in childhood and favorable cardiovascular risk in adulthood.

12. Poulton R, Moffitt TE, Silva PA. The Dunedin Multidisciplinary Health and Development Study: overview of the first 40 years, with an eye to the future. Soc Psychiatry Psychiatr Epidemiol. 2015;50:679-93. 
13. Danese A, Moffitt TE, Harrington H, et al. Adverse childhood experiences and adult risk factors for age-related disease: depression, inflammation, and clustering of metabolic risk markers. Arch Pediatr Adolesc Med. 2009;163:1135-43.

14. DeNavas-Walt C, Proctor BD, U.S. Census Bureau. Current Population Reports, P60252, Income and Poverty in the United States: 2014. Washington, DC: U.S. Government Printing Office; 2015.

15. U.S. Department of Education Office for Civil Rights. Civil Rights Data Collection: Data Snapshots (Early Childhood; School Discipline; College and Career Readiness; Teacher Equity). http://blog.ed.gov/2014/03/four-new-civil-rights-data-collection-snapshots. March 21, 2014. Accessed January 10, 2016.

16. Martin JA, Hamilton BE, Osterman MJK, Curtin, SC, Mathews, TJ, Division of Vital Statistics. Births: Final data for 2013. National vital statistics reports; vol 64 no 1. Hyattsville, MD: National Center for Health Statistics; 2015.

17. Wilmot KA, O'Flaherty M, Capewell S, Ford ES, Vaccarino V. Coronary heart disease mortality declines in the United States from 1979 through 2011: evidence for stagnation in young adults, especially women. Circulation. 2015;132:997-1002.

18. Case A, Deaton A. Rising morbidity and mortality in midlife among white non-Hispanic Americans in the 21st century. Proc. Natl. Acad. Sci. U.S.A. 2015;112:15078-83.

19. Patton GC, Coffey C, Cappa C, et al. Health of the world's adolescents: a synthesis of internationally comparable data. Lancet. 2012;379:1665-75.

20. Barker DJ. The fetal and infant origins of adult disease. BMJ. 1990;301:1111.

21. Barker DJ, Bull AR, Osmond C, Simmonds SJ. Fetal and placental size and risk of hypertension in adult life. BMJ. 1990;301:259-62. 
22. Ingelfinger JR, Nuyt AM. Impact of fetal programming, birth weight, and infant feeding on later hypertension. J Clin Hypertens. 2012;14:365-71.

23. Leon DA, Lithell HO, Vâgerö D, et al. Reduced fetal growth rate and increased risk of death from ischaemic heart disease: cohort study of 15000 Swedish men and women born 1915-29. BMJ. 1998;317:241-5.

24. Rich-Edwards JW, Colditz GA, Stampfer MJ, et al. Birthweight and the risk for type 2 diabetes mellitus in adult women. Ann Intern Med. 1999;130:278-84.

25. Christophersen ER, VanScoyoc SM. Treatments that work with children: Empirically supported strategies for managing childhood problems (2nd ed.). Washington, DC: American Psychological Association; 2013.

26. Institute of Medicine (IOM) and National Research Council (NRC). Strategies for scaling effective family-focused preventive interventions to promote children's cognitive, affective, and behavioral health: Workshop summary. Washington, DC: The National Academies Press; 2014.

27. U.S Department of Health \& Human Services, Administration for Children \& Families. Home Visiting Evidence of Effectiveness. http://homvee.acf.hhs.gov/implementations.aspx. Accessed January 10, 2016.

28. Olds DL, Eckenrode J, Henderson CR, et al. Long-term effects of home visitation on maternal life course and child abuse and neglect. Fifteen-year follow-up of a randomized trial. JAMA. 1997;278:637-43.

29. Olds DL, Kitzman HJ, Cole RE, et al. Enduring effects of prenatal and infancy home visiting by nurses on maternal life course and government spending: follow-up of a 
randomized trial among children at age 12 years. Arch Pediatr Adolesc Med. $2010 ; 164: 419-24$.

30. Olds DL, Robinson J, Pettitt L, et al. Effects of home visits by paraprofessionals and by nurses: age 4 follow-up results of a randomized trial. Pediatrics. 2004;114:1560-8.

31. Olds DL, Kitzman H, Knudtson MD, Anson E, Smith JA, Cole R. Effect of home visiting by nurses on maternal and child mortality: results of a 2-decade follow-up of a randomized clinical trial. JAMA Pediatr. 2014;168:800-6.

32. Dishion TJ, Stormshak E. Intervening in children's lives: An ecological, family-centered approach to mental health care. Washington, DC: APA Books; 2007.

33. Dishion TJ, Brennan LM, Shaw DS, McEachern AD, Wilson MN, Jo B. Prevention of problem behavior through annual family check-ups in early childhood: intervention effects from home to early elementary school. J Abnorm Child Psychol. 2014;42:343-54.

34. Lunkenheimer ES, Dishion TJ, Shaw DS, et al. Collateral benefits of the Family CheckUp on early childhood school readiness: Indirect effects of parents' positive behavior support. Dev Psychol. 2008;44:1737-52.

35. Dishion TJ, Nelson SE, Kavanagh K. The Family Check-Up with high-risk young adolescents: preventing early-onset substance use by parent monitoring. Behav Ther 2003;34:553-71.

36. Van Ryzin MJ, Nowicka P. Direct and indirect effects of a family-based intervention in early adolescence on parent-youth relationship quality, late adolescent health, and early adult obesity. J Fam Psychol. 2013;27:106-16. 
37. Smith JD, Montaño Z, Dishion TJ, Shaw DS, Wilson MN. Preventing weight gain and obesity: indirect effects of the family check-up in early childhood. Prev Sci. 2015;16:40819.

38. Webster-Stratton C, Reid MJ, Hammond M. Treating children with early-onset conduct problems: intervention outcomes for parent, child, and teacher training. J Clin Child Adolesc Psychol. 2004;33:105-24.

39. Menting AT, Orobio de Castro B, Matthys W. Effectiveness of the Incredible Years parent training to modify disruptive and prosocial child behavior: a meta-analytic review. Clin Psychol Rev. 2013;33:901-13.

40. Brotman LM, Dawson-McClure S, Huang K-Y, et al. Early childhood family intervention and long-term obesity prevention among high-risk minority youth. Pediatrics. 2012;129:e621-8.

41. Lundahl B, Risser HJ, Lovejoy MC. A meta-analysis of parent training: moderators and follow-up effects. Clin Psychol Rev. 2006;26:86-104.

42. Reyno SM, McGrath PJ. Predictors of parent training efficacy for child externalizing behavior problems--a meta-analytic review. J Child Psychol Psychiatry. 2006;47:99-111.

43. Gardner F, Connell A, Trentacosta CJ, Shaw DS, Dishion TJ, Wilson MN. Moderators of outcome in a brief family-centered intervention for preventing early problem behavior. J Consult Clin Psychol. 2009;77:543-53.

44. Gardner F, Hutchings J, Bywater T, Whitaker C. Who benefits and how does it work? Moderators and mediators of outcome in an effectiveness trial of a parenting intervention. J Clin Child Adolesc Psychol. 2010;39:568-80. 
45. McCurdy K, Daro D, Anisfeld E, et al. Understanding maternal intentions to engage in home visiting programs. Child Youth Serv Rev. 2006;28:1195-1212.

46. Borowsky IW, Mozayeny S, Ireland M. Brief psychosocial screening at health supervision and acute care visits. Pediatrics. 2003;112:129-33.

47. Pai AL, Patino-Fernandez AM, McSherry M, et al. The psychosocial assessment tool (PAT2.0): Psychometric properties of a screener for psychosocial distress in families of children newly-diagnosed with cancer. J Pediatr Psychol. 2008;33:50-62.

48. Evers-Szostak M. Integration of behavioral health care services in pediatric primary care settings. In: Maruish ME, editor. Handbook of psychological assessment in primary care settings. Mahwah, NJ: Erlbaum; 2000. p. 93-114. 